\title{
Role of bone in glucose metabolism
}

Bone is well known to influence systemic glucose homeostasis, principally via osteocalcin and insulin. However, additional mechanisms are thought to exist, but are as yet unknown. New research now suggests that cellular glucose metabolism in osteoblasts, via hypoxia signalling in bone, affects whole-body glucose homeostasis.

While investigating the role of the hypoxia signalling pathway in osteogenic cell recruitment, Christa Maes and colleagues noted that the adult mice with skeletal deletion of the hypoxia signalling pathway component von Hippel-Lindau (encoded by $\mathrm{Vhl}$ ) were much leaner than their control littermates, leading the researchers to investigate the relationship between bone and systemic energy homeostasis. They began by measuring levels of osteocalcin, which is known to influence glucose homeostasis. "We found levels of osteocalcin to be extremely low - while this would be expected to lead to hyperglycaemia and a fat phenotype, we observed exactly the opposite in our mice: they were hypoglycaemic with increased glucose tolerance, and were lean," explains Maes.

The researchers found that deletion of $\mathrm{Vhl}$ in osteoprogenitors, which models persistent hypoxia-inducible factor (HIF) activation, increased glycolysis and use of glucose in osteoblast lineage cells. As a result of these changes in cellular glucose metabolism, glucose uptake from the circulation to the osteoblast lineage cells was increased, reducing blood levels of glucose in the mice with Vhl deletion. These mice had increased glucose tolerance, which the researchers demonstrated was not related to the known actions of osteocalcin or insulin, but was rescued with the administration of a glycolysis inhibitor.

Maes and colleagues conclude that excessive glycolysis in osteoblast lineage cells that is induced by hypoxia signalling increases use of glucose in the skeleton, thus reducing systemic levels of glucose. These findings could have implications for the development of treatments for bone and metabolic disorders.

Claire Greenhill

ORIGINAL ARTICLE Dirckx, N. et al. Vhl deletion in osteoblasts boosts cellular glycolysis and improves global glucose metabolism. J. Clin. Invest. https://doi.org/10.1172//Cl97794 (2018) 